\title{
Polimorfismo -308 del gen TNFa y su expresión local en lesiones preinvasivas y cáncer de cuello uterino en el estado Zulia, Venezuela.
}

\author{
Eilyn Carolina Castro Barrios ${ }^{1}$, Tatiana Pardo Govea ${ }^{1}$, Yenddy Carrero Castillo ${ }^{1}$, \\ José Miguel Quintero Ferrer ${ }^{1}$, Yanira Sánches Caridad ${ }^{1}$, Raúl Enrique Posada Bonilla ${ }^{2}$, \\ Mercedes Fernández Mestre ${ }^{3}$ y Freddy Alaña Piña ${ }^{4}$ \\ ${ }^{1}$ Laboratorio de Genética Molecular, Instituto de Investigaciones Genéticas, Facultad \\ de Medicina, LUZ. Maracaibo, Venezuela. \\ ${ }^{2}$ Universidad del Zulia, Facultad de Medicina, Posgrado de Medicina Interna. Hospital \\ General del Sur "Dr. Pedro Iturbe". Maracaibo, Venezuela. \\ ${ }^{3}$ Laboratorio de Fisiopatología, Instituto de Medicina Experimental, IVIC. Caracas, \\ Venezuela. \\ ${ }^{4}$ Servicios Médicos Odontológicos de la Universidad del Zulia. Maracaibo, Venezuela. \\ Palabras clave: cáncer de cuello uterino; $\mathrm{TNF} \alpha$; polimorfismo -308 del gen $T N F \alpha$; \\ neoplasia intraepitelial cervical.
}

Resumen. El Factor de Necrosis Tumoral Alfa (TNF $\alpha)$, es una citocina proinflamatoria; su expresión es regulada a nivel transcripcional y se han asociado algunos polimorfismos con la progresión de cáncer de cuello uterino (CCU). El objetivo de este estudio fue caracterizar el polimorfismo -308 del gen $T N F \alpha$ y su expresión local en lesiones preinvasivas y CCU. Se analizaron muestras de ADN de sangre periférica y biopsias de cuello uterino de 8 mujeres sanas y 33 con lesiones cervicales; el $\mathrm{ADN}$ se genotipificó mediante PCR-RFLP, empleando enzimas de restricción ( $\mathrm{Ncol}$ ) y, para su expresión en tejidos, se utilizó marcaje inmunohistoquímico. El polimorfismo -308 del gen $T N F \alpha$ se encontró en Equilibrio de Hardy-Weimberg tanto en casos como en controles. El genotipo más frecuente fue el A/A (60,6\%), mientras el genotipo $\mathrm{A} / \mathrm{G}$ representó el 39,4\% del total de pacientes con lesión y no se detectó genotipo G/G; así mismo, se encontró con mayor frecuencia el alelo A, 80,3\%, mientras que el alelo G presentó una frecuencia del 19,7\%. La expresión de TNF $\alpha$ por número de células positivas en tejidos fue heterogénea, observándose diferencias estadísticamente significativas entre el grupo inflamatorio y NIC II $(p<0,045)$. No existe asociación estadísticamente significativa entre el alelo A del polimorfismo -308 del gen $T N F \alpha(\mathrm{OR}=1,53 ; p=0,585)$ con el CCU, así como entre el genotipo $\mathrm{A} / \mathrm{A}$ y el CCU, pero para aclarar su papel en la carcinogénesis se necesitan realizar más estudios cuyo tamaño de la muestra sea mayor.

Autor de correspondencia: Eilyn C. Castro B. Instituto de Investigaciones Genéticas. Facultad de Medicina. Universidad del Zulia. Maracaibo, Venezuela. Teléfonos: 0412-7682707/0424-6446103. Correo electrónico: eilyncastrob@gmail.com 


\title{
Polymorphism -308 of TNFa gene and its local expression in preinvasive lesions and cervical cancer in Zulia state, Venezuela.
}

\author{
Invest Clin 2020; 61 (4): 335-348
}

Key words: cervical cancer; $\mathrm{TNF} \alpha$; -308 polymorphism of $T N F \alpha$ gene; cervical intraepithelial neoplasia.

\begin{abstract}
Cervical cancer (CC) is a public health problem worldwide, and it represents the first cause of mortality in Venezuelan women between 25 to 44 years of age. The tumor necrosis factor alpha (TNF $\alpha)$ is a proinflammatory cytokine. Its expression is at the transcriptional level and some polymorphisms have been associated with the progression of some types of cancer, among these, the CG. The objective of the present study was to characterize the -308 polymorphism of the TNF $\alpha$ gene and its local expression in preinvasive lesions and CC. Peripheral blood DNA samples and cervical biopsies from 8 healthy women and 33 with cervical lesions were analyzed, DNA was genotyped by PCR-RFLP using restriction enzymes (NcoI), and immunohistochemical labeling was used for its expression in tissues. The -308 polymorphism of the TNF- $\alpha$ gene was found in Hardy-Weimberg equilibrium for both, individuals and lesions, as well as for healthy individuals. It was found that the most frequent genotype was AA (60.6\%), while the genotype $\mathrm{A} / \mathrm{G}$ accounted for $39.4 \%$ of the total patients with lesion and no $\mathrm{G} / \mathrm{G}$ genotype was detected. TNF expression is by cellular cells in heterogeneous tissues, statistically significant differences were observed between the inflammatory group and CIN II $(p<0.045)$. The results reveal an association between the A allele of the -308 polymorphism of the TNF $\alpha$ gene and the development of preinvasive lesions, with an $\mathrm{OR}=1.538$, with a confidence interval of $95 \%$ ranging from $0.32-7.26$ with a $\mathrm{p}=0.58$.
\end{abstract}

Recibido: 25-06-2020 Aceptado: 28-11-20200

\section{INTRODUCCIÓN}

El cáncer de cuello uterino constituye un problema de salud pública mundial (1). El carcinoma de células escamosas de cuello uterino es una de las neoplasias malignas más comunes en el sistema reproductor femenino (2), reportándose cerca de 570.000 nuevos casos de CCU y más de 311.000 muertes cada año (3). En 2019, hubo 13.170 nuevos casos de CCU y 4.250 nuevas muertes en Estados Unidos, según las estadísti- cas de cáncer (4). En Latinoamérica representa la tercera causa de muerte por cáncer (5), mientras que en Venezuela el cáncer de cuello uterino ocupa unos de los primeros lugares de mortalidad por cáncer en el género femenino, reportándose 1.387 muertes para el año 2013 (6) e incrementándose a 1.563 para el año 2014 (7). En el estado Zulia, se reportaron 206 muertes durante el año 2014, cifra que representa el 13,1\% del total de muertes por esta causa, siendo el estado con la mayor tasa de mortalidad regis- 
trada con respecto al resto del país (7). La incidencia de esta patología se ha asociado a diversos factores de riesgo que predisponen a las mujeres al desarrollo de este tipo de cáncer (1), tales como edad temprana de la primera relación sexual, múltiples compañeros sexuales, edad temprana del primer embarazo, compañeros sexuales promiscuos, bajo estado socioeconómico, infecciones vaginales y cervicales, infección por el virus del papiloma humano (VPH), herpes genital (VHS), virus de inmunodeficiencia humana (VIH), inmunosupresión, prostitución, hábito tabáquico, anticonceptivos orales (ACO), enfermedades de transmisión sexual (ETS), entre otros (1). La presencia de estos factores, puede condicionar la aparición de la enfermedad, la cual puede manifestarse con la presencia de lesiones displásicas a nivel del cuello uterino en diferentes grados y evolucionar o no a una lesión maligna e invasiva (1). Aunque, los hallazgos señalan el VPH como un factor de riesgo importante (3), no es causa suficiente para malignidad, ya que gran número de mujeres infectadas con el VPH nunca desarrollaron lesiones cervicales (8). Esto puede sugerir la implicación de los antecedentes genéticos en la transformación maligna de células epiteliales a cáncer de cuello uterino (9). La respuesta inmunitaria a nivel del cuello uterino es compleja, principalmente debido a su participación en los eventos reproductivos. La producción in situ de citocinas reviste mayor importancia que la sistémica y puede determinar diferentes efectos; así ha observado que, de acuerdo al perfil de citocinas, es posible obtener efectos proinflamatorios, inflamatorios y quimiotácticos (10). Esta respuesta local puede ser del tipo Th1, que incluye interleuquina 2 (IL-2), factor de necrosis tumoral alfa (TNF $\alpha)$, e interferón gamma (IFN- $\gamma$ ); o de tipo Th2, con IL-4, IL-5, IL-6, IL-10, IL-13 (1).

El Factor de Necrosis Tumoral alfa $(\mathrm{TNF} \alpha)$ es una citocina importante en la respuesta inflamatoria y en los mecanismos de defensa del individuo frente a infecciones virales, bacterianas y parasitarias. El TNF $\alpha$ tiene efectos antitumorales por medio de tres diferentes mecanismos biológicos: la necrosis hemorrágica causada por la citocina a nivel del endotelio tumoral, el efecto inmunomodulador sobre las células inmunitarias efectoras y el efecto citotóxico directo mediado por esta citocina. Sin embargo, el TNF $\alpha$ puede también tener diferentes efectos, los cuales pueden promover el crecimiento, proliferación del tumor y la metástasis $(11,12)$.

Existen diversos estudios donde se muestra una asociación entre el TNF $\alpha$ y el desarrollo de tumores, específicamente en células epiteliales de cuello uterino, pero existen muy pocos estudios donde se evalúe una posible asociación entre los niveles de TNF $\alpha$ y el grado de lesión, reportándose que el TNFa se expresa en los queratinocitos basales de cuello uterino normal, y se expresa con elevados niveles en la neoplasia intraepitelial cervical (NIC) de bajo y alto grado, aún en ausencia de VPH $(13,14)$. La expresión de TNF $\alpha$ está regulada a nivel transcripcional; en particular, el polimorfismo -308 del gen TNF $\alpha$ que consiste en la sustitución de una base G por A en la posición -308 de la región promotora del gen $T N F \alpha$ (15), encontrándose que la presencia del alelo A, que es menos común, está asociada con el incremento en los niveles de la citocina, mientras que el alelo G se asocia a una menor producción de la misma (1619). Algunos investigadores sugieren que el desarrollo de tumores puede estar relacionado a la predisposición genética del individuo de producir mayores niveles de TNF $\alpha$. Actualmente, existen estudios enfocados en los efectos de variaciones en la región promotora del gen $T N F \alpha$ y su relación con el cáncer de cuello uterino, encontrándose resultados contradictorios (9, 17, 20-23). Sin embargo, otros autores sugieren que la diferencia entre los niveles de producción de TNF $\alpha$ radica en la base genética de las poblaciones estudiadas $(23,24)$.

Vol. 61(4): 335 - 348, 2020 


\section{PACIENTES Y MÉTODOS}

Se examinaron 300 mujeres que acudieron a la consulta ginecológica del Hospital "Noriega Trigo", sede San Francisco, estado Zulia, Ambulatorio de San Jacinto y Servicios Médicos Odontológícos de la Universidad del Zulia, sede Maracaibo, estado Zulia, y solo 41 pacientes $(n=41)$ fueron seleccionadas intencionalmente, tomando como base los siguientes criterios de inclusión: que fueran mujeres nacidas en el estado Zulia, que por lo menos, uno de sus padres, tuviera el mismo lugar de nacimiento, y presentaran alteración colposcópica con evidencia histológica de displasia en el epitelio cervical. Para el grupo control, los criterios de inclusión fueron: pacientes nacidas en la región zuliana, no presentar ningún grado de lesión intraepitelial en cuello uterino, ni tener antecedentes personales de lesión en cuello uterino. Criterios de exclusión: estar embarazada, estar bajo tratamiento inmunosupresor, haber recibido tratamiento previo de las lesiones en cuello uterino, infección por el virus de inmunodeficiencia humana (VIH), estar en tratamiento hormonal, antiinflamatorio o anti-infeccioso local y/o sistémico por otras patologías genitales en los tres meses previos. A todas las pacientes se les realizó una historia clínica para recolectar datos sociodemográficos, personales, familiares, epidemiológícos y clínicos. Así mismo, cada paciente firmó un consentimiento informado para participar en la investigación aprobado por el Comité de Bioética de la Facultad de Medicina de la Universidad del Zulia, cumpliendo con las normas de Helsinki de principios éticos para las investigaciones médicas en seres humanos.

En la Sección de Inmunogenética del Laboratorio de Genética Molecular del Instituto de Investigaciones Genéticas de la Facultad de Medicina de La Universidad del $Z$ ulia, se colectó $5 \mathrm{~mL}$ de sangre periférica, de cada paciente, anticoagulada con ácido etilendiamino tetracético (EDTA) $5 \mathrm{mM} \mathrm{pH}$ 7,4 , para la extracción del $\mathrm{ADN}$ genómico, mediante la técnica combinada de extracción de ADN a partir de las técnicas Fenol/ Sevag (25) e Inorgánica (26). Posteriormente, se evaluó la calidad e inteǵridad del ADN de cada muestra, en un espectrofotómetro (Beckmam DU 7500) y también se confirmó a través de una electroforesis en gel de agarosa al 1\%, en buffer TBE1X a $100 \mathrm{~V}$, por un tiempo de 15 a 20 minutos, coloreando con bromuro de etidio y se observó en un transiluminador de UV, verificando así la presencia del ADN genómico. Una vez extraído el ADN y verificado su concentración, calidad e integridad, se realizó la Reacción en Cadena de la Polimerasa (PCR), para lo cual se utilizó enzimas de restricción ( $\mathrm{Ncol})$, para su genotipificación. Igualmente, se tomaron muestras de tejido de cuello uterino en los servicios de ginecología, con personal médico calificado. Se tomaron dos muestras de biopsia bajo colposcopia; una se fijó en formalina al 10\% para el análisis anatomopatológico, siguiendo los criterios de Richard (27), y la otra se incluyó en el componente OCT (Tissue Tek, Miles Inc Diaǵnostic, Kankakee, IL, USA) y se congelaron en hielo seco y acetona $\left(\mathrm{a}-70^{\circ} \mathrm{C}\right)$ para determinar la expresión de TNF $\alpha$ a nivel celular mediante pruebas de inmunofluorescencia. De las biopsias congeladas incluidas en Tissue Tek (componente OCT) se realizaron cortes de $4 \mu \mathrm{m}$ por cada tejido, utilizando un criostato Shandon ${ }^{\mathrm{TM}}$ $\left(\right.$ ThermoScientific $\left.{ }^{\mathrm{TM}}\right)$. Posteriormente se fijaron con acetona fría durante 5 minutos y se lavaron por 2 minutos con buffer fosfato salino (PBS) $\mathrm{pH} 7,4$, se incubaron durante una hora en cámara húmeda a $37^{\circ} \mathrm{C}$ con el anticuerpo monoclonal primario correspondiente al TNF (TNF- $\alpha$ : (AS1) Santa Cruz Biotechnology, Inc) previa dilución 1:500 y se lavaron con PBS pH 7,4. Para la localización se empleó un segundo anticuerpo de unión a Ig G kappa de ratón marcado con ficoeritrina (m-IgGk BP-PE Santa Cruz Biotechnology, Inc) el cual se diluyó previamente 1:500 y se incubaron a $37^{\circ} \mathrm{C}$ durante media hora en cámara húmeda, y se lavaron con PBS transcurrido el tiempo de incubación. Para el control 
de expresión se subcultivaron $10^{-} 6$ células Hela (ATCC@ CCL-2) por pozo en placas de 6 pocillos con medio DMEM, suplementado con $10 \%$ de suero fetal bovino, $100 \mu \mathrm{g} / \mathrm{mL}$ de penicilina y $100 \mathrm{U} / \mathrm{mL}$ de estreptomicina a $37^{\circ} \mathrm{C}, 5 \%$ de $\mathrm{CO}_{2}$ y atmósfera húmeda, por 24 horas sobre cover slip, seguidamente se fijaron con paraformaldehído $1 \%$ por $15 \mathrm{mi}$ nutos. Se empleó la técnica de inmunocitoquímica para el marcaje de $\mathrm{TNF} \alpha$, empleando el anticuerpo (TNF- $\alpha$ : (AS1) Santa Cruz Biotechnology, Ine) previa dilución 1:500. Para la localización se empleó un segundo anticuerpo de unión a IǵG kappa de ratón marcado con ficoeritrina (m-IgGк BP-Alexa 488® Santa Cruz Biotechnology, Inc) el cual se diluyó previamente 1:500 y se incubo a $37^{\circ} \mathrm{C}$ durante media hora en cámara húmeda, se lavó con PBS transcurrido el tiempo de incubación, posteriormente las células fueron montadas sobre portaobjetos con Aqueous Mounting (Ultra Cruz), para su visualización se utilizó un microscopio de fluorescencia Leica-DMi8 (Leica Microsystems, Alemania). El control negativo estuvo representado por IgG de ratón del mismo isótopo al anticuerpo primario. Una vez realizadas las tinciones, las muestras se secaron en oscuridad y se permontaron con UltraCruz ${ }^{\circledR}$ Aqueous Mounting Medium with DAPI para evitar la pérdida de fluoreseencia y se observaron al microscopio con epifluorescencia Leica DMi8, empleando los filtros DAPI (358-461nm) y Texa Red (595- $615 \mathrm{~nm})$ con una magnificación de 630X. Las imágenes microscópicas se capturaron en fotografías digitales a través del software Leica Application Suite (LAS) X que permite el análisis de imágenes, además de la cuantificación de la intensidad de la fluorescencia (positividad) por medio de un valor obtenido de la relación de pixeles totales de la fotografía y los pixeles del área considerada como positiva.

Análisis Estadístico. Las técnicas utilizadas para el análisis de los datos se aplicaron de acuerdo a las variables medidas: variables cualitativas (polimorfismo -308 del gen TNF $\alpha$ y grados de lesión de cuello uterino); variable cuantitativa (niveles de TNF $\alpha$ ). Con base en esto se aplicó, en cuanto al manejo descriptivo de los datos, medidas de tendencia central como la media y de dispersión como la Desviación Standard. Los niveles de expresión de TNF $\alpha$ en las muestras de tejido estudiadas se analizaron mediante el análisis de la varianza (ANOVA) y la relación del polimorfismo -308 del gen $T N F \alpha$ con la expresión del TNF $\alpha$ en tejido de cuello uterino se llevó a cabo mediante la prueba t de Student para variables independientes, empleando el paquete estadístico SPSS 17.0 y Graphap prisma 7.0. La probable asociación entre el polimorfismo -308 del gen TNF $\alpha$ y los niveles de expresión se realizó a través de chi cuadrado y Odds Ratio (OR), con un intervalo de confianza (IC) del 95\%, empleando el paquete estadístico SPSS 17.0. Las frecuencias alélicas y genotípicas observadas se evaluaron según lo esperado bajo la hipótesis del equilibrio de Hardy-Weinberǵ, utilizando el programa de genética poblacional Arlequin 3.11.

\section{RESULTADOS}

Se analizaron y procesaron 41 muestras de pacientes que cumplieron los eriterios de inclusión para esta investigación y, de acuerdo a las características clínicas, se organizaron en cinco grupos según su diaǵnóstico, de las cuales el grupo NIC I fue el más prevalente, con un $36,6 \%$, seguido de los grupos, NIC II y NIC III, con $17,1 \%$ en cada caso. El grupo conformado por pacientes con lesión inflamatoria a nivel de cuello uterino representó un $9,7 \%$ en la incidencia del total de casos. El grupo control represento el 19,5\% (Tabla I).

El rango de edad de las pacientes participantes en este estudio fue de 18 a 61 años. $\mathrm{Al}$ agrupar la muestra de acuerdo al grupo etario, se observó que la mayoría de las participantes tenían entre 25 y 44 años de edad, siendo este grupo el más afectado, dato que coincide con las edades de mortalidad en cáncer de cuello uterino en nuestro país (8).

Al determinar las frecuencias alélicas y genotípicas en los grupos estudiados se 
TABLA I

DISTRIBUCIÓN DE FREGUENCIAS SEGÚN EL NÚMERO DE CASOS PARA CADA GRUPO DE ESTUDIO.

\begin{tabular}{lcc} 
GRUPOS & $\mathrm{N}^{\circ}$ de casos & $\%$ \\
Control & 8 & 19,5 \\
Inflamatorio & 4 & 9,7 \\
NIC I & 15 & 36,6 \\
NIC II & 7 & 17,1 \\
NIC III/Ca in situ & 7 & 17,1 \\
Total & 41 & 100 \\
\hline
\end{tabular}

NIC, Neoplasia Intraepitelial Cervical. Ca, carcinoma.

observó que el alelo A fue el más frecuente, presente en los casos con lesión en un $80,3 \%$ del total de los casos con lesión (inflamatoria, NIC I,NIC II, NIC III/Ca in situ), mientras que el alelo $\mathrm{G}$, en un $19,7 \%$, siendo el grupo NICI el que reportó mayor frecuencia del alelo A $(39,4 \%)$, seguido del grupo NIC II $(16,7 \%)$, NIC III/Ca in situ $(15,1 \%)$ y el grupo inflamatorio $(9,1 \%)$. Mientras que el alelo G, para los grupos de NIC I y NIC III, se observó en un $6,1 \%$ cada uno, NIC II el 4,2\% y el grupo inflamatorio, 3\% (Tabla II).

El genotipo más frecuente en los grupos con algún grado de lesión fue el $\mathrm{A} / \mathrm{A}$ con un $60,6 \%$ y el $\mathrm{A} / \mathrm{G}$ con un $39,4 \%$, mientras que en el grupo control los genotipos $\mathrm{A} / \mathrm{A}$ y $\mathrm{A} / \mathrm{G}$ mostraron la misma frecuencia
(Tabla III). El genotipo G/G no se encontró en la muestra estudiada. Se registró mayor frecuencia del genotipo $\mathrm{A} / \mathrm{A}$ en el grupo NIC I $(33,3 \%)$, seguido del grupo NIC II $(12,1 \%)$, NIC III/Ca in situ $(9,1 \%)$ y el grupo inflamatorio $(6,1 \%)$. Mientras que el genotipo $\mathrm{A} / \mathrm{G}$, para NIC I y NIC III reportó $12,1 \%$ cada uno, NIC II el 9,1\% y el grupo inflamatorio $6,1 \%$.

Las distribuciones genotípicas observadas en los grupos de estudio fueron concordantes con lo esperado bajo la hipótesis de equilibrio de Hardy-Weinberg $p>0,05$, no significativo. La población está en equilibrio de Hardy Weinberg, $\mathrm{X}^{2}=2,41, \mathrm{P}=0,11$.

Para conocer una posible asociación entre el polimorfismo -308 del gen TNF $\alpha$ y el desarrollo de lesiones preinvasivas de cuello uterino, se formuló una tabla de contingencia $\mathrm{X}^{2}=0,298$. Al calcular el Odds Ratio, se obtuvo, $\mathrm{OR}=1,53$ con un intervalo de confianza de $95 \%(0,32-7,26)$ y $\mathrm{p}=0,585$. Aunque estos resultados no muestran diferencias estadísticamente significativas, probablemente por el reducido tamaño de la muestra, al observar la estimación de riesǵo (Odds Ratio-OR), las pacientes que poseen el alelo A del polimorfismo -308 del gen TNF $\alpha$ tienen 1,53 veces más probabilidad de desarrollar algún grado de lesión.

Al realizar el análisis inmunohistoquímico, para determinar la expresión de TNF $\alpha$

TABLA II

FRECUENCIAS ALÉLICAS EN PACIENTES CON LESIONES INFLAMATORIAS, NEOPLASIA INTRAEPITELIAL GERVICAL Y PACIENTES CONTROL.

\begin{tabular}{|c|c|c|c|c|c|c|c|c|c|c|c|c|}
\hline \multirow[t]{4}{*}{ ALELO } & \multicolumn{10}{|c|}{ DIAGNÓSTICO } & & \\
\hline & \multirow{2}{*}{\multicolumn{2}{|c|}{$\begin{array}{c}\text { Sano } \\
\text { control }\end{array}$}} & \multicolumn{8}{|c|}{ Lesiones a Nivel Cervical } & \multirow{2}{*}{\multicolumn{2}{|c|}{$\begin{array}{c}\text { Total } \\
\text { (Lesiones) }\end{array}$}} \\
\hline & & & \multicolumn{2}{|c|}{ Inflamatorio } & \multicolumn{2}{|c|}{ NIC I } & \multicolumn{2}{|c|}{ NIC II } & \multicolumn{2}{|c|}{$\begin{array}{c}\text { NIC III/ } \\
\text { Ca in situ }\end{array}$} & & \\
\hline & $\mathrm{n}$ & $\%$ & $\mathrm{n}$ & $\%$ & $\mathrm{n}$ & $\%$ & $\mathrm{n}$ & $\%$ & $\mathrm{n}$ & $\%$ & $\mathrm{n}$ & $\%$ \\
\hline $\mathrm{A}$ & 12 & 75 & 6 & 9,1 & 26 & 39,4 & 11 & 16,7 & 10 & 15,1 & 53 & 80,3 \\
\hline G & 4 & 25 & 2 & 3 & 4 & 6,1 & 3 & 4,3 & 4 & 6,1 & 13 & 19,7 \\
\hline Total & 16 & 100 & 8 & 12,1 & 30 & 45,5 & 14 & 21 & 14 & 21,2 & 66 & 100 \\
\hline
\end{tabular}

NIC, Neoplasia Intraepitelial Cervical. Ca, carcinoma. 
en las muestras de tejidos, se analizó tanto la intensidad de la expresión de la citocina como el número de células positivas para TNF $\alpha$. Figs. 1, 2 y 3.

Las medidas descriptivas como la media y desviación estándar en la cuantificación del número de células positivas para la expresión de TNF $\alpha$ en tejidos de cuello uterino para cada grupo fueron: control (sano): $6,93 \pm 2,81$; inflamatorio: $10,85 \pm 4,107$; NIC I: $8,35 \pm 2,73$; NIC II: 5,82 \pm 1,92; NIC III: $8,31 \pm 1,75$. En la Fig. 4 , se observa la distribución de las medias en cada grupo.
Los resultados obtenidos en el análisis de la varianza (ANOVA) de la expresión de TNF $\alpha$ según el número de células positivas, se consideraron no significativos $(\mathrm{P}>0,05=$ 0,05. Sin embargo, al aplicar el test de comparación múltiple Bonferroni, se encontró diferencia significativa al comparar el grupo inflamatorio con el grupo NIC II, observándose una diferencia de medias de 5,02571 \pm 1,65641 células por campo, y una $\mathrm{p}=0,045$, con un intervalo de confianza del 95\%.

En la Fig. 5 se observan los resultados obtenidos al analizar la intensidad de la ex-

\section{TABLA III \\ FRECUENCIAS GENOTÍPICAS EN PACIENTES CON ALGÚN GRADO DE LESIÓN Y EL GRUPO CONTROL.}

\begin{tabular}{|c|c|c|c|c|c|c|c|c|c|c|c|c|}
\hline \multirow[t]{4}{*}{ GENOTIPO } & \multicolumn{12}{|c|}{ DLAGNÓSTICO } \\
\hline & \multirow{2}{*}{\multicolumn{2}{|c|}{$\begin{array}{c}\text { Sano } \\
\text { control }\end{array}$}} & \multicolumn{10}{|c|}{ Lesiones a Nivel Cervical } \\
\hline & & & \multicolumn{2}{|c|}{ Inflamatorio } & \multicolumn{2}{|c|}{ NIC I } & \multicolumn{2}{|c|}{ NIC II } & \multicolumn{2}{|c|}{$\begin{array}{l}\text { NIC III/ } \\
\text { Ca in situ }\end{array}$} & \multicolumn{2}{|c|}{$\begin{array}{c}\text { Total } \\
\text { (lesiones) }\end{array}$} \\
\hline & $\mathrm{n}$ & $\%$ & $\mathrm{n}$ & $\%$ & $\mathrm{n}$ & $\%$ & $\mathrm{n}$ & $\%$ & $\mathrm{n}$ & $\%$ & $\mathrm{n}$ & $\%$ \\
\hline $\mathrm{A} / \mathrm{A}$ & 4 & 50 & 2 & 6,1 & 11 & 33,3 & 4 & 12,1 & 3 & 9,1 & 20 & 60,6 \\
\hline $\mathrm{A} / \mathrm{G}$ & 4 & 50 & 2 & 6,1 & 4 & 12,1 & 3 & 9,1 & 4 & 12,1 & 13 & 39,4 \\
\hline Total & 8 & 100 & 4 & 12,2 & 15 & 45,4 & 7 & 21,2 & 7 & 21,2 & 33 & 100 \\
\hline
\end{tabular}

NIC, Neoplasia Intraepitelial Cervical. Ca, Carcinoma.
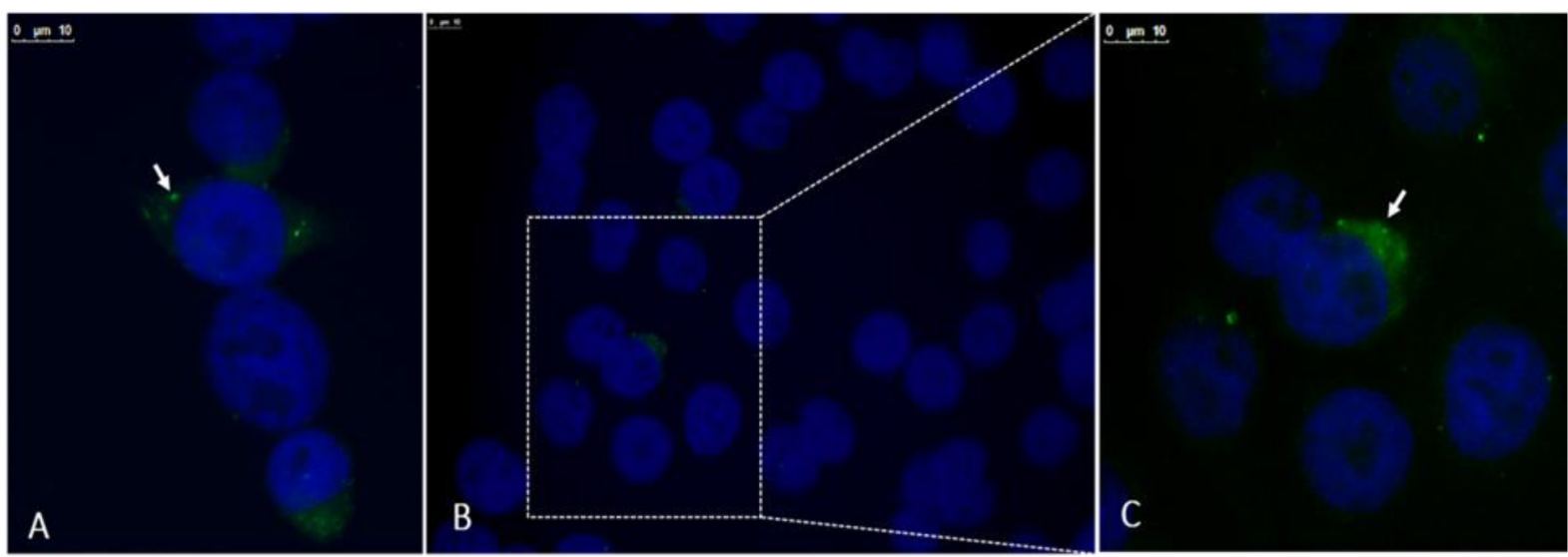

Fig. 1. Las imágenes A y B muestran expresión de TNF $\alpha$ en células Hela (ATCC® CCL-2), las cuales se incluyeron como control positivo y $\mathbf{C}$ la maǵnificación (ROI) de la imaǵen $\mathbf{B}$ en el cual se evidencia la

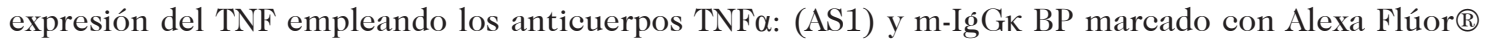
488. Maǵnificación 630X. 


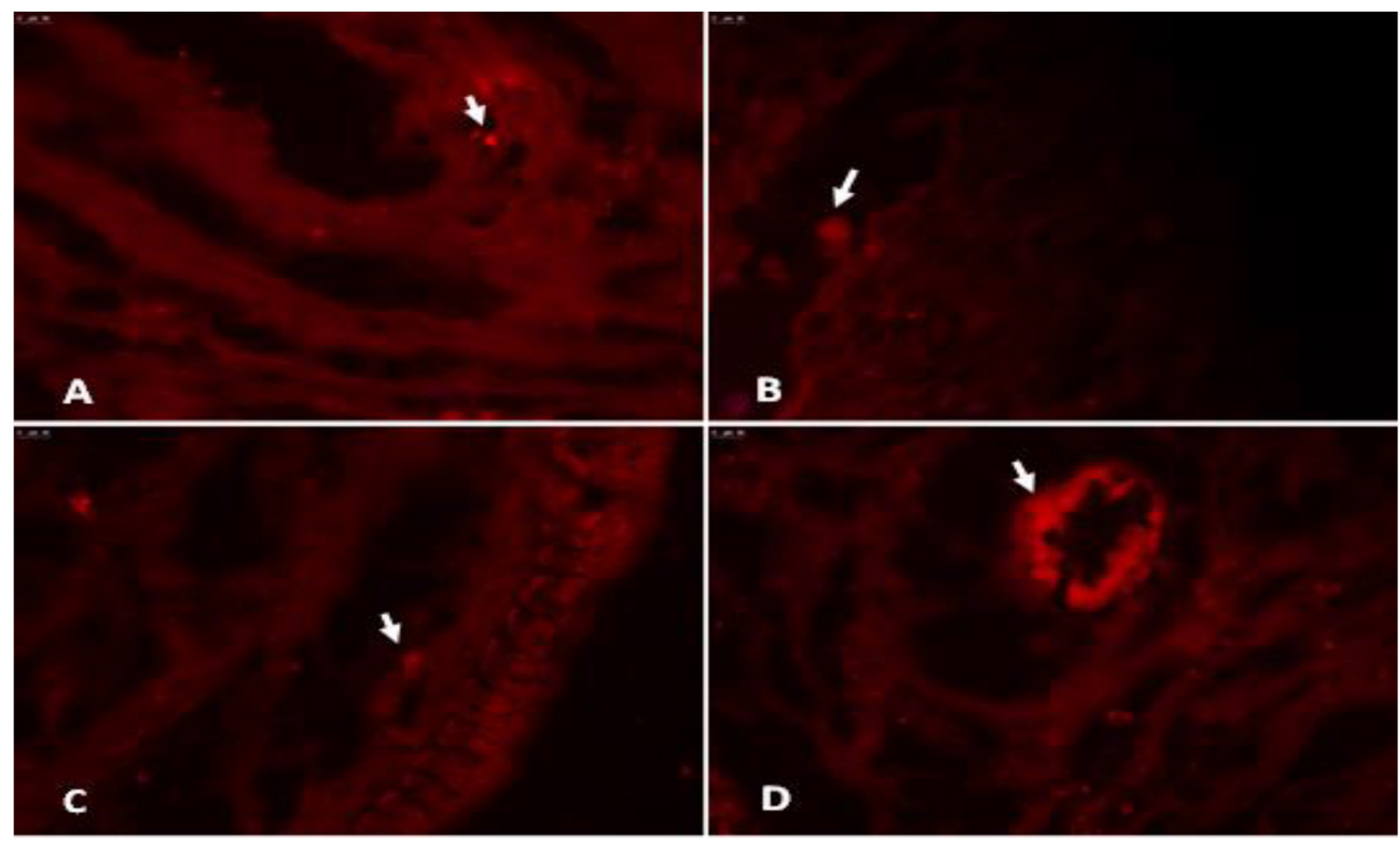

Fig. 2. Expresión de TNF $\alpha$ en pacientes sanos y con endocervicitis crónica a través de análisis inmunohistoquímico. Las imágenes A y B muestran expresión de TNFa en tejidos procedentes de pacientes sanos, mientras que $\mathbf{C}$ y $\mathbf{D}$ en tejidos de pacientes con endocervicitis crónica. Las flechas señalan células positivas para TNFa empleando los anticuerpos. Magnificación 630X.

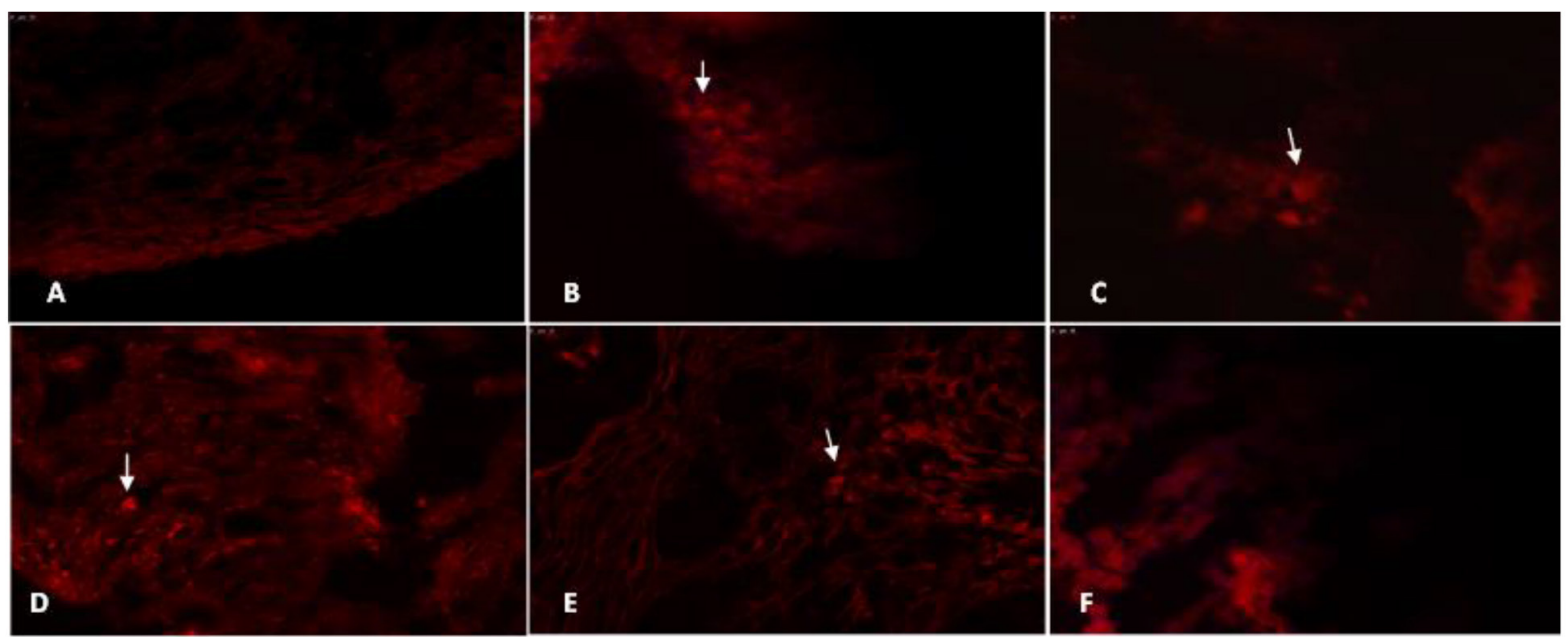

Fig. 3. Expresión de TNF $\alpha$ en tejidos con lesiones en cuello uterino a través de análisis inmunohistoquímico. Las imágenes A y $\mathbf{D}$ muestran expresión de TNF $\alpha$ en tejidos procedentes de pacientes diaǵnosticados con NIC I, B y E pacientes con NICII, mientras que $\mathbf{C}$ y $\mathbf{F}$ pacientes diagnosticados con cáncer in situ. Las flechas señalan células positivas para TNF $\alpha$ empleando los anticuerpos TNF- $\alpha$ : (AS1) y m-IǵGк BP marcado con ficoeritrina. Magnificación 630X. 


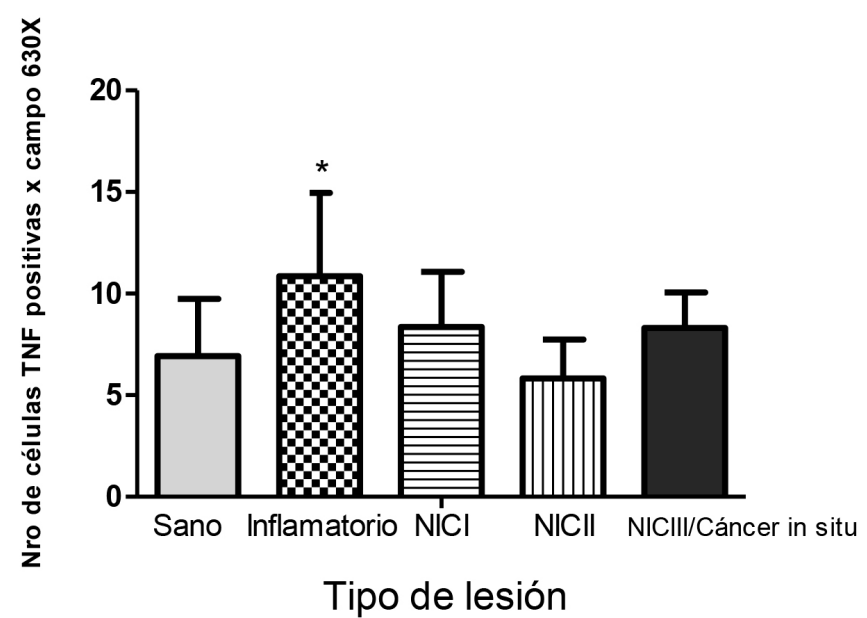

${ }^{*} \mathrm{p}<0.05$ inflamatorio Vs NICII

Fig. 4. Número de células TNF $\alpha$ positivas distribuidas de acuerdo al tipo de lesión y controles. NIC, neoplasia intraepitelial cervical. TNF, factor de necrosis tumoral.

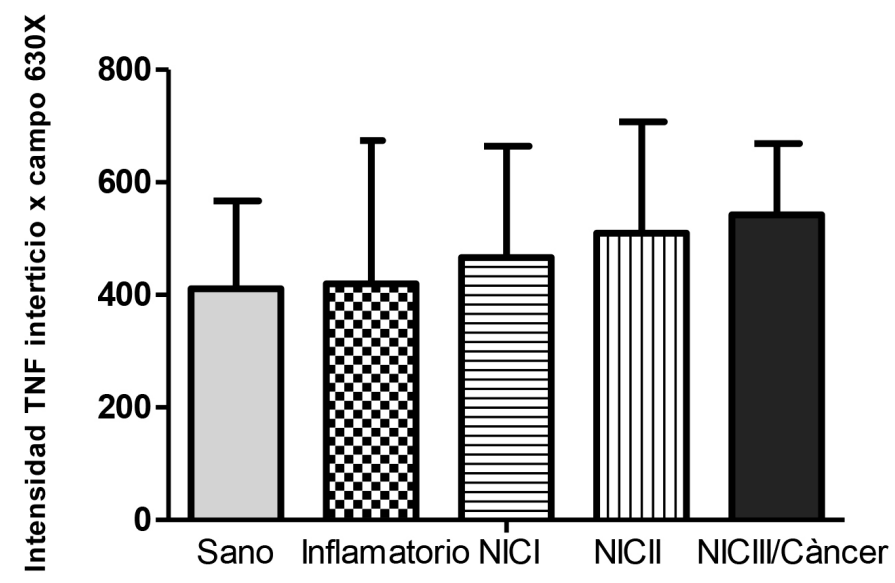

\section{Diagnóstico}

Fig. 5 Intensidad por campo de la expresión de TNFa distribuidas de acuerdo al tipo de lesión y controles.

presión en el intersticio de TNF $\alpha$ por campo en las muestras de tejido de cuello uterino. Se determinaron las siguientes medias y desviación estándar para cada grupo: control (sano): 410,835 $\pm 155,92$; inflamatorio: $419,46 \pm 254,66$, NIC I, 466,35 $\pm 198,05$, NIC II, 509,52 \pm 198,05 y NIC III/Ca in situ $542,110 \pm 126,65$. Al realizar el análisis de la varianza no se encontró diferencias significativas entre los pacientes con algún grado de lesión en cuello uterino y los pacientes sanos, ya que el valor de $\mathrm{p}$ fue mayor de 0,05. Sin embargo, se puede observar que el nivel de intensidad en la expresión de la citocina va aumentando de acuerdo la inflamación progresa a algún grado de Neoplasia Intraepitelial Cervical.

Al evaluar la posible asociación entre el polimorfismo -308 y la expresión de TNF $\alpha$ en los tejidos, a través de la prueba $\mathrm{t}$ de Stu- 
dent para muestras independientes, no se encontraron diferencias significativas en el recuento de células positivas para la citocina, y tampoco al contrastar los resultados con la intensidad del intersticio por campo. Por lo tanto, el número de células positivas por campo como la intensidad del intersticio que muestran la expresión de TNF $\alpha$, son independientes del genotipo por lo que se establece que no hay asociación entre estas variables.

\section{DISCUSIÓN}

El cáncer de cuello uterino (CCU) representa un problema de salud pública mundial, debido a su alta prevalencia y alta tasa de mortalidad. En Venezuela, es la primera causa de muerte para el género femenino en edades entre 25-64 años de edad (7). Los resultados de morbilidad observados son similares ya que las pacientes que presentaron enfermedad inflamatoria o algún grado de neoplasia intraepitelial cervical se encontraban en este grupo etario. Se ha sugerido que la presencia del polimorfismo -308 del gen $T N F-\alpha$ puede influir en la gravedad de varias enfermedades y asociarse con la aparición de cáncer de cuello uterino, pero los resultados no han sido concluyentes hasta el momento (24). En este estudio se analizaron las frecuencias alélicas y genotípicas de 41 pacientes para determinar su asociación con el desarrollo de lesiones preinvasivas y cáncer de cuello uterino. No se encontraron estudios en donde se evaluara la relación genotipo con la expresión génica en tejido de cuello uterino con o sin algún grado de lesión, por lo que los resultados del presente estudio se compararon con estudios poblacionales en donde se investigó el polimorfismo -308 del gen TNF- $\alpha$ en diversas poblaciones caucásicas, mestizas, asiáticas, entre otros.

El genotipo $\mathrm{A} / \mathrm{A}$, ha sido asociado con mayor riesgo a desarrollar distintas enfermedades y diferentes tipos de cáncer, según lo reportado para otras poblaciones del mundo (28). En este estudio, se encontró presente en un $\mathbf{5 8 , 5 \%}$, mientras que el genotipo he- terocigoto $\mathrm{A} / \mathrm{G}$ se detectó en un 41,5\% y el genotipo $\mathrm{G} / \mathrm{G}$ no se evidenció en ninguna muestra. Estos resultados demuestran que del total de casos con lesión a nivel de cuello uterino, el alelo A fue el más frecuente al analizar los resultados en los grupos con algún grado de lesión $(80,3 \%)$, frente al alelo $\mathrm{G}(19,7 \%)$, contrario a lo estudiado por Acosta y col. en poblaciones mestizas y de oriǵen caucásico del Perú (28), en donde estudiaron 135 individuos utilizando la técnica PCR RFLP encontrando que la presencia del alelo A es el alelo menos frecuente $(12,5 \%)$ en mestizos y ausente en caucásicos, el genotipo $\mathrm{A} / \mathrm{G}$ se detectó en $5,2 \%$ en mestizos de Lima y Loreto, siendo el genotipo G/G el más frecuente con un $87,5 \%$.

En el estudio realizado por Barbisan y col. (29), en una muestra de 122 pacientes y 176 controles de La Plata, Argentina, determinaron el polimorfismo -308 del gen TNF- $\alpha$ de muestras de ADN, a través de la técnica de PCR RFLP, pero no encontraron asociación entre genotipos del polimorfismo estudiado y el riesgó a desarrollar cáncer de cuello uterino, lo que concuerda con los resultados del presente estudio. Sin embargo, encontraron la presencia del genotipo A/G en un $32 \%$ de los casos (29).

Rotar y col. (30), analizaron la relación entre el polimorfismo - 308 de gen TNF $\alpha$ y NIC, en pacientes del condado Cluj-Napocaen en Rumania. Se estudiaron muestras de ADN de 78 casos de NIC y 107 controles mediante PCR-RFLP, y en todas las clases de neoplasia intraepitelial cervical, el genotipo G/G fue el que se encontró con mayor frecuencia; $\sin$ embargó, contrario a nuestros resultados, el genotipo A/A fue extremadamente raro en la población de estudio, pero sus datos sugieren una asociación entre la presencia de un alelo A y cáncer invasivo (OR $=10,8261$, IC del $95 \%=1.0748-109.0511, \mathrm{p}=0.0433)$.

Estudios en otras poblaciones como el de Haoshan y col. (31), analizaron la relación entre el TNF $\alpha-308 \mathrm{G}>\mathrm{A},-238 \mathrm{G}>\mathrm{A}$, y TNFRII-VNTR -322 y el cáncer cervical en 240 pacientes de Han, China, con cáncer cervical 
invasivo y 220 pacientes sanos (control). La genotipificación del polimorfismo -308 del gen TNF $\alpha$ se realizó mediante PCR-RFLP. El análisis estratificado de FIGO mostró que la variante menor $\mathrm{A} / \mathrm{A}$ y el $\mathrm{G} / \mathrm{A}+\mathrm{A} / \mathrm{A}$ combinado de $T N F \alpha-238 \mathrm{G}>\mathrm{A}$ y $T N F \alpha-308 \mathrm{G}>\mathrm{A}$ aumentaron el riesgó de evolución tumoral, $(\mathrm{P}=0,011, \mathrm{OR}=2,91, \mathrm{IC}$ del $95 \%=1,11-7,79$; $\mathrm{P}=0,005, \mathrm{OR}=2,79$, IC del 95\%=1,28-6,81; $\mathrm{P}=0,001, \mathrm{OR}=15,33$, respectivamente); observándose una asociación estadísticamente significativa entre el polimorfismo del TNFa y la progresión clínica del cáncer según la clasificación de la FIGO. Estos resultados sugieren un incremento en el riesgó para cáncer cervical en pacientes con genotipo $\mathrm{A} / \mathrm{A}$ y A/G (31).

Li y col. (32) correlacionaron el polimorfismo de TNF- $\alpha-308$ y la susceptibilidad al cáncer de cuello uterino en 142 pacientes con cáncer de cuello uterino y 150 controles sanos, utilizando PCR-RFLP y enzima de restricción $\mathrm{NCoI}$. Al igual que nuestros resultados, no encontraron diferencias significativas en las frecuencias genotípicas y alélicas entre el grupo de pacientes con cáncer de cuello uterino y el grupo control; sin embargo, observaron que el genotipo A/A pudiera estar asociado con el incremento del riesgo de desarrollar cáncer de cuello uterino en 1,46 veces, con un intervalo de confianza del $95 \%$ de 0,32 a 6,67. Además, encontraron que pacientes con el alelo A tuvieron cáncer cervical de alto grado de malignidad (32).

Li y col. (32), también correlacionaron 5 polimorfismos en la región promotora del gen TNFa (rs361525, rs1800629, rs1800750, rs1799964, and rs673) con la susceptibilidad a desarrollar cáncer de cuello uterino, en 946 muestras de sangre periférica de mujeres de la etnia Han de la provincia de Shandong, de las cuales 452 fueron diagnosticadas con carcinoma cervical de células escamosas y 492 pacientes controles. Las muestras fueron analizadas empleando PCR SNP`s utilizando el método de la sonda TaqMan. Estos resultados de Li y col. (32) están en contradicción con los obtenidos en el presente estudio donde hubo diferencias estadísticamente significativas entre los grupos de casos y controles para el polimorfismo rs1800629 $(\mathrm{P}<0,001)$ que tuvo una correlación significativa con CCU. La frecuencia del alelo A para el polimorfismo rs1800629 fue significativamente elevada para el cáncer cervical $(29,9 \%)$ con respecto al grupo control $(14,2 \% ; \mathrm{OR}=2,58$, IC $95 \%=1,87-3,56, \mathrm{P}<0,01)$. Los polimorfismos rs361525, rs1800629, y rs17999645 se correlacionaron significativamente con el diagnnóstico de cáncer cervical (9).

Du y col. (33), investigaron la distribución de SNP de TNF (-238 G/A, -308 G/A) e IL10 (-592 G/G, -819 G/T y - $1082 \mathrm{G} / \mathrm{A})$ y su relación con el cáncer de cuello uterino en una gran cohorte de mujeres chinas. En sus resultados reportaron para el polimorfismo -308 , una mayor frecuencia genotípica para $\mathrm{A} / \mathrm{A}$ en pacientes con cáncer de cuello uterino que en controles. En ese trabajo se encontró asociación estadísticamente significativa entre los genotipos $\mathrm{A} / \mathrm{A}$ del polimorfismo TNF-308 y los genotipos $\mathrm{C} / \mathrm{A}$ o $\mathrm{A} / \mathrm{A}$ de la IL10-592 y las pacientes con cáncer cervical ( $\mathrm{p}<0,0167)$. Además, encontraron que la combinación de las variantes $\mathrm{G} / \mathrm{A}$ del polimorfismo $T N F \alpha-308$ con la variante $\mathrm{C} / \mathrm{A}$ de la IL10-592 y la combinación G/G o G/A del polimorfismo $T N F \alpha-308$ con la variante $\mathrm{C} / \mathrm{A}$ o A/A de la IL10, tuvieron una asociación con la severidad del cáncer cervical cuando están juntos. Estos resultados sugieren que los genotipos $\mathrm{A} / \mathrm{A}$ del polimorfismo $T N F \alpha-308$ y $\mathrm{CA} / \mathrm{AA}$ de la IL10-592 CA/AA pueden incrementar la susceptibilidad para desarrollar el cáncer cervical a través de la alteración de la respuesta inmunitaria de un individuo (33). Aunque nuestros resultados no fueron estadísticamente significativos, encontramos que el genotipo $\mathrm{A} / \mathrm{A}$ presentó una frecuencia del $60,6 \%$ en pacientes con algún grado de lesión a nivel de cuello uterino.

Al determinar la asociación de la expresión de TNF $\alpha$ en tejidos con el genotipo, no hubo diferencias significativas; sin embargo, al cuantificar la intensidad por campo de la 
expresión de TNF $\alpha$ en las muestras de tejido de cuello uterino, se observó que ésta se incrementaba gradualmente desde el proceso inflamatorio a NIC I, II y III/Ca in situ para la población de estudio. Tales resultados también son contradictorios a los hallazgos reportados por Pardo en el 2005 (1) en pacientes de San Francisco, estado Zulia-Venezuela, donde no encontraron diferencias significativas a medida que aumenta el grado de lesión al observar el número de células positivas para TNF $\alpha$ mediante estudio de inmunofluorescencia indirecta en cortes de tejido cervical; sin embargo, al comparar la expresión de TNF $\alpha$ en cada uno de los grupos de lesión, con respecto al grupo control, se observó diferencias significativas con los estadios NIC I y NIC III $(\mathrm{p}<0,008)(1)$. Otro estudio similar, donde se observó el número de células positivas para TNF $\alpha$ mediante estudio de inmunofluorescencia indirecta en cortes de tejido cervical, fue el de CallejasMonsalve en el 2011 (34), en pacientes de Maracaibo y San Francisco, estado Zulia, Venezuela, el cual también mostró resultados contrarios a los obtenidos en este estudio, con diferencias significativas con los estadios NIC I y NIC III al compararlos con los grupos control $(\mathrm{p}<0,05)$, mientras que en nuestro estudio se observó diferencias signnificativas al comparar el grupo inflamatorio con NIC II $(\mathrm{p}<0,045)$.

El análisis estadístico del presente estudio demostró que no existe asociación significativa entre el alelo A del polimorfismo -308 del gen $T N F \alpha$ con el $\mathrm{CCU}(\mathrm{OR}=1,53$; $\mathrm{p}=0,585)$, así como entre el genotipo $\mathrm{A} / \mathrm{A}$ $\mathrm{y}$ el CGU, probablemente por el reducido número de la muestra. Sin embargo, cabe destacar que el alelo A fue el más frecuente $(80,3 \%)$ frente al alelo $G(19,7 \%)$, y pudiera esto representar un factor de riesgo para las pacientes que participaron en el estudio, por lo que se recomienda realizar nuevos estudios donde se amplíe el tamaño de la muestra y, de esta manera, obtener mayor información que permita comprender mejor la distribución alélica y genotípica del poli- morfismo -308 del gen $T N F \alpha$ y su relación con la predisposición al desarrollo y/o evolución de las lesiones preinvasivas a cáncer de cuello uterino.

Este estudio es inédito en el estado Zulia y Venezuela, puesto que no solo se consideraron los polimorfismos, sino también su expresión en el tejido del cuello uterino. Si bien no arrojó datos concluyentes, permitió dilucidar el papel de este factor genético en el proceso de carcinogénesis y en los procesos inflamatorios que pudiesen preceder a la lesión.

\section{REFERENCIAS}

1. Pardo T, Callejas D, Nuñez T J, Araujo M, Costa L, Pons H, Delgado M, Monsalve F. Expresión de interferon gamma (IFN $\gamma$ ), factor de necrosis tumoral alfa $(\mathrm{TNF} \alpha)$ e interleucinas 2, 4, 6 (IL-2, IL-4, IL-6) en células de neoplasias intraepiteliales de cuello uterino: Reporte preliminar. Invest Clin 2005; 46: 5-13.

2. Ma J, Cheng P, Chen X, Zhou C, Zheng W. Mining of proginosis-related genes in cervical squamous cell carcinoma immune microenvironment. Peer J 2020; 8:e9627.

3. Guo L, Hua K. Cervical Cancer: emerging immune landscape and treatment. Onco Targets Ther 2020; 13:8037-8047.

4. Siegel RL, Miller KD, Jemal A. Cancer statistics, 2019. CA: Cancer J Clin 2019; 69(1):7-34.

5. Capote Negrin LG. Epidemiology of cervical cancer in Latin America. Ecancermedicalscience 2015; 9:577.

6. Ministerio del Poder Popular para la Salud. Anuario de Mortalidad 2013. Disponible en: http://www.mpps.gob.ve/ index.php?option $=$ com_phocadownloa $\mathrm{d} \&$ view $=$ categ ory\&id=11:anuarios-demortalidad\&Itemid=915. Diciembre 2015 .

7. Ministerio de Poder Popular para la Salud. Anuario de Mortalidad 2014. Disponible en: https://www.ovsalud.org/descargas/publicaciones/documentos-oficiales/AnuarioMortalidad-2014.pdf. 2018

8. Roszak A, Misztal M, Sowinska A, Jagodzinski P. $T N F \alpha-308 \mathrm{G} / \mathrm{A}$ as risk market of cervical cancer progression in the Polish population. Mol Diaǵn Ther 2015; 19:53-57. 
9. Li X, Yin G, Li J, Wu A, Yuan Z, Liang J, Sun $Q$. The correlation between TNF- $\alpha$ promoter gene polymorphism and genetic susceptibility to eervical cancer. Technol Cancer Res Treat 2018; 17:1533033818782793.

10. Carrero Y, Callejas D, Mindiola R, Pardo T, Atencio R, Araujo M. Respuesta inmunitaria en cuello uterino: Infección por VPH y neoplasia intraepitelial cervical. Acta Científica Estudiantil 2006; 4(3):125-136.

11. Szlosarek P, Charles K, Balkwill F. Tumor necrosis factor- $\alpha$ as a tumor promoter. Eur J Cancer 2006; 42(6):745-750.

12. Shishodia S, Majumdar S, Banerjee S, Aggarwal B. Ursolic acid inhibits nuclear factor- $\mathrm{kB}$ activation induced by carcinogenic agents through suppression of І $\mathrm{I} \mathrm{B} \alpha$ kinase and p65 phosporylation: correlation with down-regulation of cyclooxygenase 2 , matrix metalloproteinase 9, and cyclin D1. Cancer Res 2003; 63(15):4375-4383.

13. Mota F, Rayment S, Chong A, Singer A, Chain B. The antigen-presenting environment in normal and human papillomavirusrelated premalignant cervical epithelium. Clin Exp Immunol 1999; 116(1):33-40.

14. Tjiong $M$, Van der Vange $N$, Ten Kate $F$, Tjong $\mathbf{A}$, Schegget $\mathbf{J}$, Burger $\mathbf{M}$. Increased IL-6 and IL-8 levels in cervicovaginal secretions of patients with eervical cancer. Gynecol Oncol 1999; 73(2):285-291.

15. Yi F, Shi X, Pei X, Wu X. Tumor necrosis factor-alpha-308 gene promoter polymorphism associates with survival of eancer patients: A meta-analysis. Medicine (Baltimore) 2018;97(45):e13160.

16. Deshpande A, Nolan J, White P, Valdez Y, Hunt W, Peyton C, Wheeler C. TNF-a promoter polymorphisms and susceptibility to human papillomavirus 16-associated cervical cancer. J Infect Dis 2005; 191(6):969-976.

17. Tjiong $\mathbf{M}$, Van der Vange $\mathbf{N}$, TerSchegget $\mathbf{J}$, Burger M, Ten Kate F, Out T. Cytokines in cervicovaginal washing fluid from patients with eervical neoplasia. Cytokine 2001; 14(6):357-360.

18. Kroeger K, Carville $K$, Abraham L. The -308 tumor necrosis factor-alpha promoter polymorphism effects transcription. Mol Immunol 1997; 34(5):391-399.

19. Chen G, Wilson $R$, Wang $S$, Zheng $H$, Walker J, McKillop J. Tumor necrosis fac- tor alpha (TNF- $\alpha$ ) gene polymorphism and expression in pre-eclampsia. Clin Exp Immunol 1996; 104(1):154-159.

20. Calhoun E, McGovern R, Janney C, Cerhan J, Iturria S, Smith D, Gostout B, Persing D. Host genetic polymorphism analysis in cervical eancer. Clin Chem 2000; 48(8):12181224.

21. Stanczuk G, Sibanda E, Tswana S, Bergostrom S. Polymorphism at the -308-promoter position of the tumor necrosis factor alpha (TNF-alpha) gene and cervical cancer. Int J Gynecol Cancer 2003; 13(2):148-153.

22. Ghaderi M, Nikitina L, Peacock C, Hjelmstrom P, Hallmans G, Wiklund F, Lenner P, Blackwell J, Dillner J, Sanjeevi C. Tumor necrosis factor a-11 and DR15-DQ6 ( $\mathrm{B} * 0602)$ haplotype increase the risk for cervical intraepithelial neoplasia in human papillomavirus 16 seropositive women in Northern Sweden. Epidemiol Biomarkers Prev 2001; 9(10):1067-1070.

23. Chagaas $B$, Lima R, Paiva Júnior S, Silva R, Cordeiro M, Silva N, Batista M, Silva A, Gurgel A, Freitas A. Significant association between IL10-1082/-819 and TNF-308 haplotypes and the susceptibility to cervical carcinogenesis in women infected by human papillomavirus. Cytokine 2019;113:99-104.

24. Vandana G, Constant D, Hoffman M, Williamson A. The allelic distribution of -308 tumor necrosis factor-alpha gene polymorphism in South African women with eervical eancer and control women. BMC Cancer 2006; 6: 24.

25. Miller A, Dykes D, Polesky H. A simple salting out procedure for extraction DNA from human nucleated cells. Nucl Acid Res 1988; 16(3): 1215.

26. Ye S, Dhillon S, Ke X, Collins A, Day I. An efficient procedure of genotyping single nucleotide polymorphism. Nucleic Acids Res 2001; 29(17):88-96.

27. Richard R. Influence of diagnostic and therapeutic procedures on the distribution of cervical intraepithelial neoplasias. Cancer 1996; 19(11): 1635-1638.

28. Acosta O, Solano L, Oré D, Salazar A, Sandoval J, Fujita R. Polimorfismo -308 G/A en la región promotora del gen factor de necrosis tumoral alfa (TNFA) en diferentes subpoblaciones peruanas. Revista Horizonte Médico 2010, 10:1. 
29. Barbisan G, Pérez L, Contreras A, Golijow $\mathrm{C}$, TNF- $\alpha$ and IL-10 promoter polymorphisms, HPV infection, and eervical cancer risk. Tumor Biol 2012; 33:1549-1556. Doi 10.1007/s13277-012-0408-1.

30. Rotar I, Mureșan D, Radu P, Petrișor F, Apostol S, Tane M, Butuza C, Stamatian F. TNF- $\alpha 308 \mathrm{G} / \mathrm{A}$ polymorphism and eervical intraepithelial neoplasia. Anticancer Res 2014; 34: 373-378.

31. Haoshan L, Jun T, Hailing C, Hongxia $Z$, Dongli Z. Association of tumor necrosis factor alpha polymorphisms with cervical cancer in a Chinese population. Int $\mathrm{J}$ Clin Exp Pathol 2016; 9(2):2200-2207.

32. Li L, Liu J, Liu C, Lu X. The correlation between TNF- $\alpha-308$ gene polymorphism and susceptibility to cervical cancer. Oncol Lett 2018; 15(5):7163-7167.
33. Du GH, Wang JK, Richards JR, Wang JJ. Genetic polymorphisms in tumor necrosis factor alpha and interleukin-10 are associated with an increased risk of cervical cancer. Int Immunopharmacol 2019; 66:154161.

34. Callejas D. Caracterización de la Respuesta Inmunitaria Inflamatoria en el Cuello Uterino en Pacientes con Displasias de Cérvix. Tesis doctoral, Universidad de Alcalá de Henares, 2011. Disponible en: http:// dspace.uah.es/dspace/bitstream/handle/10017/14301/Tesis\%20Doctoral\%20 Diana\%20Callejas.pdf? sequence $=1$. 